\title{
Scattering of Surface States at Step Edges in Nanostripe Arrays
}

\author{
F. Schiller, ${ }^{1}$ M. Ruiz-Osés, ${ }^{2}$ J. Cordón, ${ }^{2}$ and J. E. Ortega ${ }^{1,2}$ \\ ${ }^{1}$ Donostia International Physics Center, Paseo Manuel Lardizabal 4, E-20018 Donostia-San Sebastián, Spain \\ ${ }^{2}$ Departamento de Física Aplicada I and Centro Mixto CSIC/UPV, Universidad del País Vasco, \\ Plaza de Oñate 2, E-20018 Donostia-San Sebastián, Spain
}

(Received 19 April 2005; published 4 August 2005)

\begin{abstract}
Surface states of noble metal surfaces split into Ag-like and $\mathrm{Cu}$-like subbands in stepped $\mathrm{Ag} / \mathrm{Cu}$ nanostripe arrays. The latter self-assemble by depositing Ag on vicinal $\mathrm{Cu}(111)$. Ag-like states scatter at nude step edges in Ag stripes, leading to umklapp bands, quantum size effects, and peak broadening. By contrast, Ag stripe boundaries become transparent to $\mathrm{Cu}$-like states, which display band dispersion as in flat $\mathrm{Cu}(111)$. We find a linear relationship between the quantum size shift and peak broadening that applies in a variety of stepped systems, revealing the complex nature of step barrier potentials.
\end{abstract}

DOI: 10.1103/PhysRevLett.95.066805

Surface electron scattering at atomic steps is of key importance to understand fundamental physical properties of zero-dimensional (0D) and one-dimensional (1D) crystalline nanostructures and arrays self-assembled on solid surfaces, like quantum dots, atomic wires, or nanostripes [1-4]. In such fine systems the two-dimensional (2D) surface plane contains two chemically different phases, i.e., the nanostructure and the supporting substrate, with phase boundaries that are frequently defined by atomic step edges. Consequently, surface electron scattering at steps determines electronic interactions between phases [5], gives rise to size effects in lateral nanostructures [6], and even influences the equilibrium structure in a stepped surface [7]. Despite their importance, the study of freeelectron-like surface states in two-phase nanostructure arrays with atomic steps as phase boundaries has never been addressed. Here we investigate this subject using a simple model system, namely, a 1D striped metallic array.

It is generally believed that the step potential arises from the smear out of the surface charge around the step edge that leads to a step dipole [8]. However, its effective interaction with extended surface states can vary significantly. Steps frequently behave as poorly reflective and strongly repulsive potentials for surface electrons [6,9], but they have been observed to become weak, transmitting barriers for high step densities in vicinal surfaces [1012], or to behave as refractive interfaces in insulating layer boundaries [5]. Here we study step scattering in metallic nanostripe arrays analyzing quantum size effects and lifetime broadening of their surface states. As shown in Fig. 1, a 1D array of 1-monolayer (ML)-thick Ag nanostripes are self-assembled by step flow growth of $\mathrm{Ag}$ on vicinal $\mathrm{Cu}(111)$. With $0.6 \mathrm{ML}$, the scanning tunneling microscopy (STM) image in Fig. 1(a) displays 1-ML-thick, elongated $\mathrm{Ag}$ stripes with their triangular moiré reconstruction, which alternate, in a local scale, with clean $\mathrm{Cu}(111)$ terraces. The side view of the system is schematically shown in Figs. 1(b) and 1(c) before and after deposition. Ag stripes develop faster in the perpendicular direction of the steps, filling up some of the terraces while leaving
PACS numbers: 73.20.At, 68.37.Ef, 73.40.-c

others empty. As a consequence, a step array with double step is formed at the surface, and the step dipole potential is transferred from $\mathrm{Cu}$ steps in the clean surface to doubleatomic step edges in Ag stripes (marked $A$ in Fig. 1). As also depicted in Fig. 1(c), we find that surface states in this nanostripe array split into $\mathrm{Ag}$-like and $\mathrm{Cu}$-like states, with different scattering properties at step boundaries. Our data indicate weak scattering at $\mathrm{Cu}$ steps decorated with $\mathrm{Ag}(B$ boundaries in Fig. 1), as expected for a locally reduced dipole moment, as well as strong downhill or uphill asymmetry. Downhill, Ag states scatter strongly at $A$ boundaries, as proved by the step-lattice umklapp bands, the quantum size effects, and the surface peak broadening with respect to flat, infinite $\mathrm{Ag}$ monolayers. Uphill, $\mathrm{Cu}$ states are insensitive to this dipole barrier, and hence they propagate freely in the surface plane like in flat $\mathrm{Cu}(111)$. A comprehensive analysis of surface states in different types of stepped arrays reveals a universal proportionality between quantum shift and peak broadening by scattering at metallic step edges, which reflects the complex nature of the step potential.

Angle resolved photoemission spectroscopy (ARPES) was performed at $150 \mathrm{~K}$ with a high-resolution angle
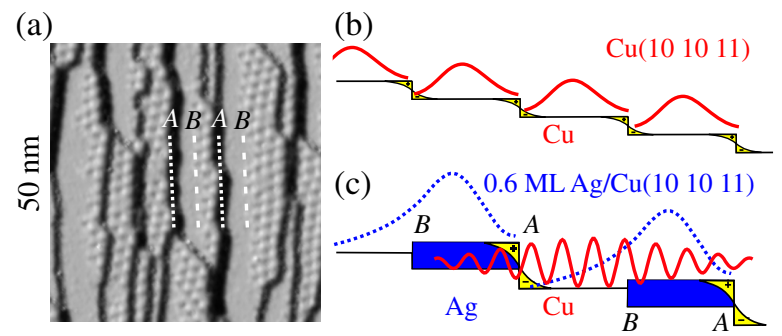

FIG. 1 (color online). (a) STM image of $0.6 \mathrm{ML} \mathrm{Ag}$ on $\mathrm{Cu}(101011)$, showing one-monolayer-thick $\mathrm{Ag}$ nanostripes that fill up substrate terraces as depicted in (c). The dipole potential is transferred from $\mathrm{Cu}$ steps in the clean surface (b) to Ag stripe edges (marked $A$ ). Surface states split into Ag-like (dotted) states that scatter at $A$ boundaries in the stripe, and $\mathrm{Cu}-$ like (solid) that are insensitive to the dipole potential. 
resolved hemispherical analyzer SCIENTA 200. The samples were illuminated by a high-intensity He discharge lamp with monochromatized photons of $h \nu=21.2 \mathrm{eV}$ (He I $\alpha$ ), $h \nu=40.8 \mathrm{eV}$ (He II $\alpha$ ), and $h \nu=48.4 \mathrm{eV}$ $(\mathrm{He}$ II $\beta$ ). Energy and angular resolutions were set to $30 \mathrm{meV}$ and $0.3^{\circ}$, respectively. The stepped crystal is $\mathrm{Cu}(101011)$, i.e., vicinal to $\mathrm{Cu}(111)$ with $2.7^{\circ}$ miscut towards [1 112$], d=43 \AA$ step-lattice constant, and $\{100\}$-like packing at steps. The clean surface displays well-ordered, linear step arrays along the [1110] direction [13], leading to sharp split spots in the low energy electron diffraction pattern and umklapp surface state bands in ARPES (see Fig. 2). The terrace width distribution (TWD) measured by STM is $\sigma=\Delta d / d=0.25$ [13], i.e., better than the ones obtained for vicinal $\mathrm{Cu}(111)(\sigma>$ $0.3)$ with $\{111\}$-like step edges $[11,14]$. Ag is deposited at $150 \mathrm{~K}$ and shortly postannealed to $300 \mathrm{~K}$. The $150 \mathrm{~K}$ deposition and the relatively large value of $d$ give rise to step flow growth of Ag, which otherwise induces substrate faceting [15,16]. Ag grows faster perpendicular to the steps, resulting in 1-ML-thick $\mathrm{Ag}$ stripes of $d=43 \AA$ width and finite length that fill up substrate terraces. At 0.6 ML, this leads to alternating $\mathrm{Ag}$ and $\mathrm{Cu}$ nanostripes with half of the substrate step density, although the periodic array is defined only in a local scale, as shown in Fig. 1(a).

Figure 2 contains photoemission intensity plots taken at different photon energies, which show the surface band dispersion along $k_{\|}$in the direction perpendicular to the step array for $\mathrm{Cu}(101011)$ and $0.6 \mathrm{ML} \mathrm{Ag} / \mathrm{Cu}(101011)$. In the latter, distinct $\mathrm{Cu}$-like (band bottom at $E_{F}-435 \mathrm{meV}$ ) and $\mathrm{Ag}$-like $\left(E_{F}-207 \mathrm{meV}\right)$ bands can be distinguished, similar to those found in $\mathrm{Ag} / \mathrm{Cu}(111)$ [17,18]. Both $\mathrm{Ag}$ and $\mathrm{Cu}$ states are, respectively, originated at Ag-covered and free surface patches. By tuning $h \nu$ one probes the

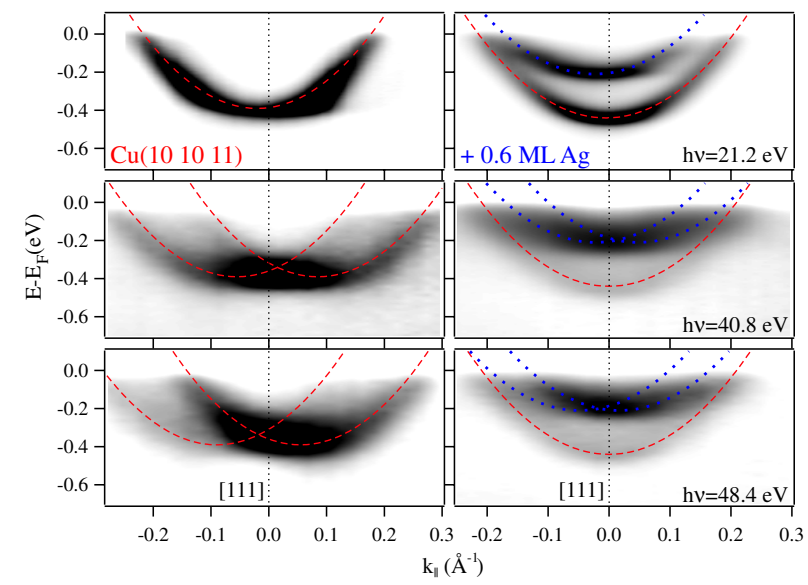

FIG. 2 (color online). Photoemission intensity plots showing umklapp surface bands by the step lattice in $\mathrm{Cu}(101011)$ (left) and $\mathrm{Ag}$-like and $\mathrm{Cu}$-like surface bands for $0.6 \mathrm{ML}$ $\mathrm{Ag} / \mathrm{Cu}(101011)$ structure (right hand side) at $h \nu=21.2$, 40.8 , and $48.4 \mathrm{eV}$. The $k_{\|}$axis runs perpendicular to the steps with $k_{\|}=0$ marking the [111] direction. spectral composition of the surface state in the perpendicular direction [10]. Despite the reduced thickness of the $\mathrm{Ag}$ stripe, the intensity changes in Fig. 2 reveal a distinct, Aglike character of the $\mathrm{Ag}$ state wave function. The intensity is similar for $\mathrm{Ag}$ and $\mathrm{Cu}$ states at $h \nu=21.2 \mathrm{eV}$, but becomes much higher for the $\mathrm{Ag}$ band at photon energies of 40.8 and $48.4 \mathrm{eV}$, i.e., close to the $L$ point resonance in the $\mathrm{Ag}$ band structure at $48 \mathrm{eV} \mathrm{[19].} \mathrm{The} \mathrm{same} \mathrm{resonance}$ would be tuned with $h \nu=67 \mathrm{eV}$ in the case of $\mathrm{Cu}$ [11]. Thus, the Ag-like surface state in Fig. 2 is not an interface state like that of $\mathrm{NaCl}$-covered $\mathrm{Cu}(111)$ [5], which also leads to a shifted band but still retains its $\mathrm{Cu}$ nature.

The scattering at the step array is directly visualized in the photon-energy-dependent umklapp bands of Figs. 2 and 3 for $\mathrm{Cu}$ states in the clean surface and $\mathrm{Ag}$ states in nanostripes, but not for $\mathrm{Cu}$-like states in the 0.6 ML array. Photoemission umklapps display photon-energydependent $k_{\|}$-position and intensity variations. Umklapp bands of similar intensity are particularly well observed at $h \nu=40.8$ and $48.4 \mathrm{eV}$. In Fig. 2 the weak parabolic envelopes for $\mathrm{Cu}$ and $\mathrm{Ag}$ bands are marked by dashed and dotted lines, respectively. In clean $\mathrm{Cu}(1010$ 11) the two bands are shifted by $\Delta k_{\|}=0.145 \AA^{-1}$, which exactly corresponds to the step-lattice vector $2 \pi / d$, with $d=$ $43 \AA$ A. In the $0.6 \mathrm{ML} \mathrm{Ag} / \mathrm{Cu}(101011)$ plots we can observe two Ag bands shifted by $\Delta k_{\|}=(0.07 \pm 0.01) \AA^{-1}$, which correspond to $2 \pi / d$ umklapps, with $d \approx 90 \AA$. This distance is similar to the average superstructure periodicity $d=86 \AA$ (Ag stripe + Cu terrace) of Fig. 1, indicating that the step scattering is mainly produced downhill at double-step $A$ boundaries. Yet the Ag band umklapps in the right panels of Fig. 2 appear significantly broadened along $k_{\|}$compared to $\mathrm{Cu}$ bands in the left panels, as expected from the reduced coherence of the $\mathrm{Ag} / \mathrm{Cu}$ superstructure. Figure 3 shows the energy distribution curves (EDC's) that correspond to the photoemission images taken at $h \nu=21.2 \mathrm{eV}$. Here we can better observe how the surface state umklapp (schematically shown by vertical dashed and dotted lines) passes from the $\mathrm{Cu}$ to the $\mathrm{Ag}$ surface state after 0.6 ML Ag deposition. We also observe that the Ag band actually displays a very reduced dispersion, like quasi-1D quantum well bands in vicinal surfaces $[11,13]$. By contrast to $\mathrm{Ag}, \mathrm{Cu}$ nanostripe states clearly define a strongly dispersive single band in both the EDC spectra of Fig. 3 and the image plots of Fig. 2. Thus, the existence of umklapp bands in the present case is explained by surface state scattering at steps, and not to final state photoelectron interference provoked by the step array [20], since that would affect both $\mathrm{Cu}$ and $\mathrm{Ag}$ states alike.

Figure 4 compares the surface state spectra at the band minima for different $\mathrm{Ag}$ coverage on $\mathrm{Cu}(111)$ and $\mathrm{Cu}(101011)$. Both samples have been mounted together, allowing a peak-to-peak comparison for spectra measured under the same experimental conditions. In particular, we can accurately determine in each case the upwards energy shift $(\Delta E)$ and peak broadening $(\Delta \omega)$ with respect to the 


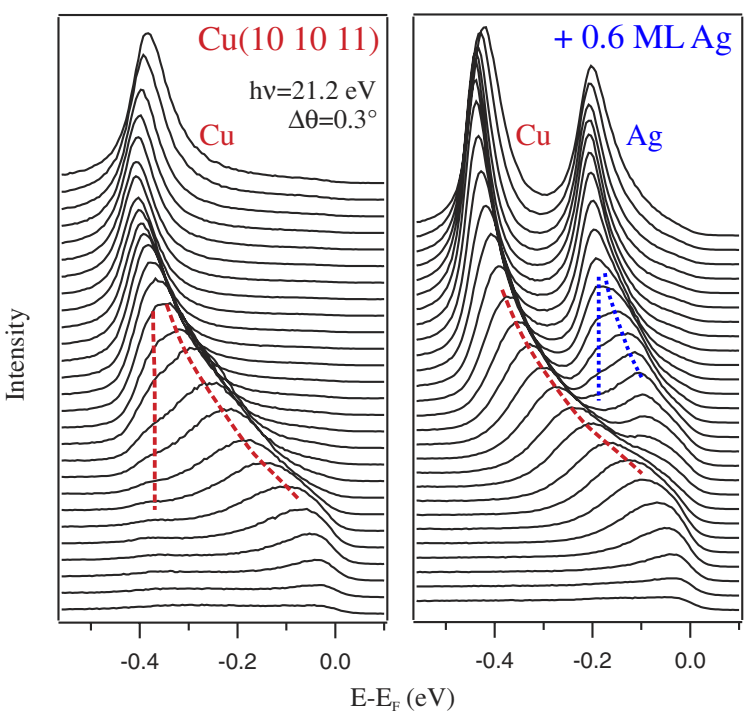

FIG. 3 (color online). Band dispersion for $\mathrm{Cu}(1010$ 11) and $0.6 \mathrm{ML} \mathrm{Ag} / \mathrm{Cu}(101011)$ corresponding to Fig. $2(h \nu=$ $21.2 \mathrm{eV}$ ). The umklapp is transferred from the $\mathrm{Cu}$ band in the clean surface to the Ag peak in the 0.6 ML nanostripe array, which also displays weaker dispersion.

flat surface, which are the common signatures of the surface state scattering at step edges $[10,11,14] . \Delta E$ reflects the quantum size effect by total or partial confinement within terraces, whereas the change in the width $\Delta \omega$ is explained as due to a reduced photohole lifetime by absorption at step edges or by overlap and mixing of surface states and bulk states on terraces $[10,11,14,21]$. The striking observation of Fig. 4 is that energy shift and broadening affect all surface state peaks, except the $\mathrm{Cu}$ state in the 0.6 ML nanostripe array. Indeed, both the surface state in clean $\mathrm{Cu}(101011)$ and the $\mathrm{Ag}$ peaks for 0.6 and $1 \mathrm{ML}$ shift by $\Delta E=30,14$, and $8 \mathrm{meV}$, and broaden by $\Delta \mathrm{FWHM}=$ $\Delta \omega=35,17$, and $5 \mathrm{meV}$, respectively. By contrast, the $\mathrm{Cu}$ peak of the 0.6 ML spectrum has almost shifted back to the position $(\Delta E=3 \mathrm{meV})$ of the surface state in flat $\mathrm{Cu}(111)$, and has also narrowed to the same peak width $(\Delta \omega=0 \mathrm{meV})$. Since the $\mathrm{Cu}$ peak disperses freely along $k_{\|}$(Fig. 3, right), we conclude that surface states of $\mathrm{Cu}$ nanostripes in Fig. 1(c) do not significantly scatter at $A$ or $B$ stripe boundaries. In the uphill direction $\mathrm{Cu}$ stripe states ignore Ag-like double-step edges. In the downhill direction, $\mathrm{Ag}$-decorated $\mathrm{Cu}$ steps become almost transparent; i.e., the step barrier is quenched without introducing absorptive scattering, which is, by contrast, the case of Fe or $\mathrm{CO}$ decorating $\mathrm{Cu}$ steps $[22,23]$.

The energy shift $\Delta E$ gives an estimate of the strength $U_{0} b$ of the step potential. Assuming a Kronig-Penney model of periodic deltalike barriers [10], the relationship between $\Delta E$ and $U_{0} b$ can shortly be written as

$$
\Delta E=\frac{2 \hbar^{2}}{m^{*}} \frac{1}{d^{2}}\left[\tan ^{-1}\left(\frac{q_{0}}{q}\right)\right]^{2}
$$

where $q_{0}=\left(m^{*} / \hbar^{2}\right) \times U_{0} b$ and $q=\sqrt{\left(2 m^{*} / \hbar^{2}\right) \Delta E}$. For

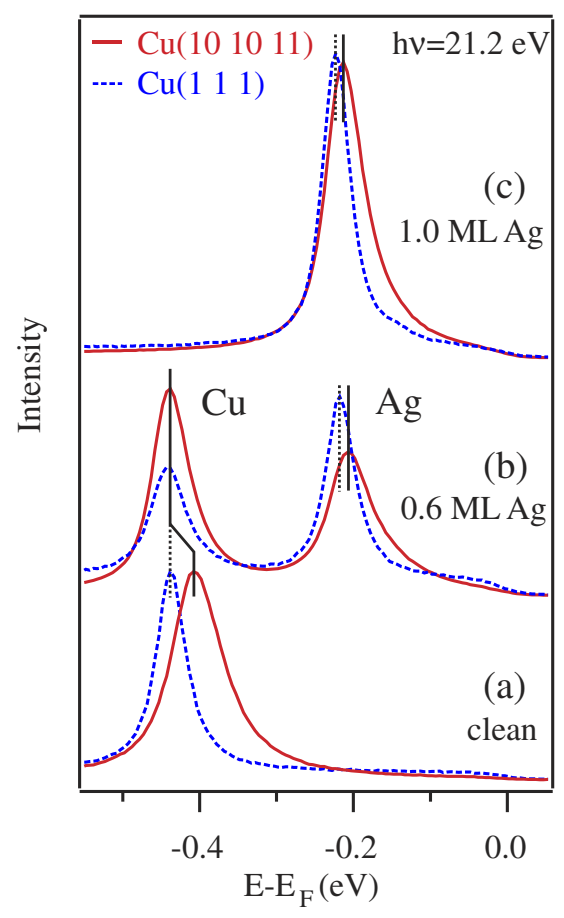

FIG. 4 (color online). PE spectra taken at the surface band bottom for (a) $\mathrm{Cu}(101011)$ and $\mathrm{Cu}(111)$, and covered with (b) $0.6 \mathrm{ML} \mathrm{Ag}$ and (c) $1 \mathrm{ML} \mathrm{Ag}$. Peak shift and broadening with respect to the respective flat systems are the evidence for scattering at steps. It is observed in Ag-like peaks and clean $\mathrm{Cu}(1010$ 11) surface states, and it is absent in $\mathrm{Cu}$-like states of the striped structure (0.6 ML).

both $\mathrm{Ag}$ and $\mathrm{Cu}$ states of the clean and $\mathrm{Ag}$-covered $\mathrm{Cu}(101011) m^{*}=0.41 m_{e}$ was used. Using Eq. (1), for single steps in $\mathrm{Cu}(101011)(d=43 \AA, \Delta E=30 \mathrm{meV})$ and $1 \mathrm{ML} \mathrm{Ag} / \mathrm{Cu}(101011)(d=43 \AA, \Delta E=8 \mathrm{meV})$ we, respectively, obtain $U_{0} b=2.9$ and $0.8 \mathrm{eV} \AA$. Thus, the Ag monolayer exhibits a reduced strength compared to the step barrier potential of $\mathrm{Cu}(111)$ and $\mathrm{Au}(111)$ vicinal surfaces with similar values of $d[10]$, reflecting the different chemical environment. For the $\mathrm{Ag}$ peak in the $0.6 \mathrm{ML}$ nanostructure the effective confinement region $d$ of the wave function is unknown. However, the umklapp bands in Figs. 2 and 3 suggest a negligible scattering at $B$ like boundaries, and hence we can assume $d=86 \AA$. In this case the observed $\Delta E=14 \mathrm{meV}$ corresponds, within the accuracy of the experiment, to the energy shift of the infinite potential well of size $d=86 \AA$, i.e., $(\hbar \pi)^{2} /(2 m \times$ $\left.d^{2}\right)=12 \mathrm{meV}$. This result agrees with the reduced dispersion of the Ag peak observed in Fig. 3, and suggests strong scattering at double-step edges and effective 1D confinement of $\mathrm{Ag}$ states in the $\mathrm{Ag} / \mathrm{Cu}$ nanostructure of Fig. 1.

Regardless of the system (surface, monolayer, or nanostripe array), one can qualitatively observe a close relationship between peak broadening $\Delta \omega$ and quantum shift $\Delta E$ in all Ag-like and $\mathrm{Cu}$-like states of Fig. 4. This is analyzed in detail in Fig. 5, where we plot $\Delta E$ versus $\Delta \omega$ measured from the spectra in Fig. 4. We have also 


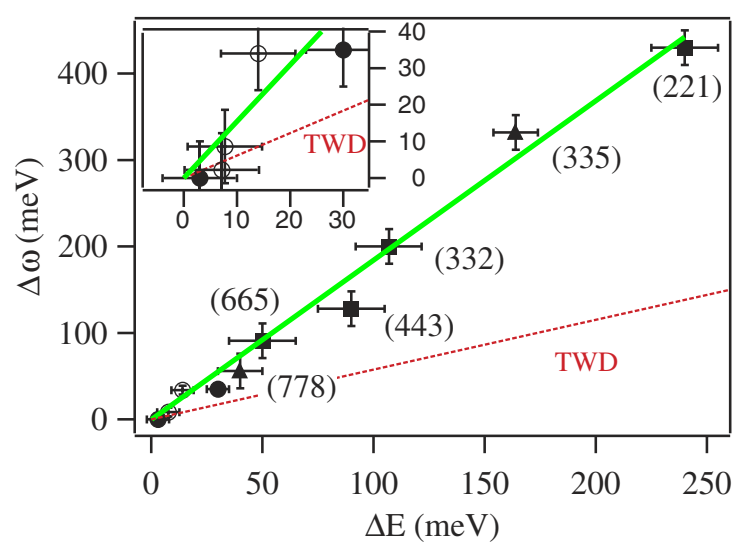

FIG. 5 (color online). Energy shift versus peak broadening (FWHM) with respect to the corresponding flat systems for Ag-like (open circles) (inset) and $\mathrm{Cu}$-like surface states (filled circles). Black squares and triangles are data measured in $\mathrm{Cu}(111)$ vicinals in Refs. [7,11,13,14].

added the case of $2 \mathrm{ML} \mathrm{Ag} \mathrm{(} \Delta E=7 \mathrm{meV}, \Delta \omega=2 \mathrm{meV})$, as well as recent high-resolution ARPES data measured from photoemission spectra of vicinal $\mathrm{Cu}(111)$ taken from Refs. [7,11,13,14]. The area enlarged in the inset of Fig. 5 contains all data points deduced from the analysis of Fig. 4. A single solid line fits the whole set of data (slope $1.8)$ and intersects the origin. The linear $\Delta \omega(\Delta E)$ relationship appears as a universal property, since there is no significant dependence on the nature of the step edge, i.e., $\mathrm{Ag}$ or $\mathrm{Cu}$, double or single. Peak width and quantum shift are minima for low densities and weak barriers, as in $\mathrm{Ag} / \mathrm{Cu}(101011)$, and both increase for stronger barriers, as in $\mathrm{Cu}(101011)$, or higher step densities, as in $\mathrm{Cu}(221)$.

Notice that $\Delta w$ in Fig. 5 contains both photohole lifetime and TWD broadening contributions. The latter can be estimated from Eq. (1) by differentiating $\Delta E$ and $d$. We obtain $\Delta \omega_{\mathrm{TWD}}=f\left(q_{0} / q\right) \sigma \Delta E$, where $\sigma=\Delta d / d$, and $f\left(q_{0} / q\right)$ varies from 2 to 1 from infinite to small step barriers, respectively. Assuming a generic $\sigma=0.3$, the dashed line in Fig. 5 represents the maximum value $\Delta \omega_{\text {TWD }}=0.6 \Delta E$, i.e., less than $30 \%$ of the total change in $\Delta \omega$, which is then explained by photohole decay mainly. The proportionality between $\Delta E$ and $\Delta \omega$ observed in Fig. 5 therefore suggests that the lifetime contribution to $\Delta \omega$ depends on the step density $(d)$ and the barrier strength $\left(q_{0}\right)$ in a similar way as $\Delta E$ in Eq. (1). Such an equation could thus be generalized to also account for $\Delta \omega$ by considering complex step barriers. That would describe a local, absorptive step scattering scenario, rather than the elastic decay on terraces due to surface or bulk state mixing, as suggested previously $[10,11]$. The latter, which can be due to direct overlap but also to umklapping with superlattice vectors, has, indeed, been shown to contribute with less than $10 \mathrm{meV}$ to the total peak width in $\mathrm{Cu}(111)$ [24]. Nonetheless, absorptive scattering at complex step barriers is also connected to elastic decay into bulk states, as shown by Hörmandinger and Pendry for rows of scatterers on $\mathrm{Cu}(111)$ [25]. There, the peak broadening is found proportional to the barrier strength, but also to the physical overlap between the surface state wave function and the step edge. Interestingly, such overlap decreases away from the band bottom and becomes minimum at superlattice zone boundaries, where wave functions are antibonding states of the step array. In clean $\mathrm{Cu}(101011)$ we can, indeed, observe a decrease in $\Delta \omega$ as we move away from the band minimum, further proving its connection to local absorption at steps.

This work has been funded by the Spanish Ministerio de Educación y Ciencia (FIS2004-06490-C03-03, MAT2002-11975-E), the DAAD/MCyT exchange program, the Basque government Grant No. BFI04.313.R2 and, as part of the European Science Foundation EUROCORES Programme SONS, by the EC Sixth Framework Programme. We thank A. Mugarza and P. M. Echenique for a critical reading of the manuscript.

[1] V. A. Shchukin and D. Bimberg, Rev. Mod. Phys. 71, 1125 (1999).

[2] M. Pratzer et al., Phys. Rev. Lett. 87, 127201 (2001).

[3] P. Gambardella et al., Nature (London) 416, 301 (2002).

[4] J. N. Crain et al., Phys. Rev. B 69, 125401 (2004).

[5] J. Repp, G. Meyer, and K.-H. Rieder, Phys. Rev. Lett. 92, 036803 (2004).

[6] L. Diekhöner et al., Phys. Rev. Lett. 90, 236801 (2003).

[7] F. Baumberger et al., Phys. Rev. Lett. 92, 016803 (2004).

[8] X. Y. Wang, X. J. Shen, and R. M. Osgood, Jr., Phys. Rev. B 56, 7665 (1997).

[9] L. Bürgi, O. Jeandupeux, A. Hirstein, H. Brune, and K. Kern, Phys. Rev. Lett. 81, 5370 (1998).

[10] A. Mugarza and J. E. Ortega, J. Phys. Condens. Matter 15, S3281 (2003).

[11] F. Baumberger et al., Phys. Rev. Lett. 92, 196805 (2004).

[12] M. Hansmann, J. I. Pascual, G. Ceballos, H.-P. Rust, and K. Horn, Phys. Rev. B 67, 121409(R) (2003).

[13] J.E. Ortega et al., New J. Phys. 7, 101 (2005).

[14] F. Baumberger, T. Greber, and J. Osterwalder, Phys. Rev. B 64, 195411 (2001).

[15] A. R. Bachmann, A. Mugarza, J. E. Ortega, and S. Speller, Phys. Rev. B 64, 153409 (2001).

[16] J. Lobo et al., Phys. Rev. Lett. 93, 137602 (2004).

[17] A. Bendounan et al., Phys. Rev. B 67, 165412 (2003).

[18] F. Schiller, J. Cordón, D. Vyalikh, A. Rubio, and J.E. Ortega, Phys. Rev. Lett. 94, 016103 (2005).

[19] T. C. Hsieh, P. John, T. Miller, and T.-C. Chiang, Phys. Rev. B 35, 3728 (1987).

[20] A. M. Shikin et al., Phys. Rev. Lett. 93, 146802 (2004).

[21] R. Eder and H. Winter, Phys. Rev. B 70, 085413 (2004).

[22] S. Shiraki, H. Fujisawa, M. Nantoh, and M. Kawai, Phys. Rev. Lett. 92, 096102 (2004).

[23] F. Baumberger, T. Greber, B. Delley, and J. Osterwalder, Phys. Rev. Lett. 88, 237601 (2002).

[24] C. Corriol et al. (unpublished).

[25] G. Hörmandinger and J.B. Pendry, Phys. Rev. B 50, 18607 (1994). 